\title{
Avian-Inspired Passive Perching Mechanism for Robotic Rotorcraft
}

\author{
Courtney E. Doyle, Justin J. Bird, Taylor A. Isom, C. Jerald Johnson, Jason C. Kallman, Jason A. Simpson, \\ Raymond J. King, Jake J. Abbott, and Mark A. Minor
}

\begin{abstract}
Flying robots capable of perch-and-stare are desirable for reconnaissance missions. Current solutions for perchand-stare applications utilize various methods to create an aircraft that can land on a limited set of surfaces that are typically horizontal or vertical planes. This paper presents a bio-inspired concept that allows for passive perching on cylindrical-type surfaces. The prototype provides compliant gripping through the use of an underactuated foot. A mechanism inspired by songbird anatomy is integrated that utilizes rotorcraft weight as a way to passively actuate the foot. Successful perching trials on two rods of differing diameters were performed and are discussed. The purpose of this initial design is to act as a proof of concept for the mechanical action of the mechanism; our results demonstrate that passive perching can be achieved through the integration of underactuated gripping with mechanismgenerated mechanical advantage.
\end{abstract}

\section{INTRODUCTION}

Perch-and-stare describes a maneuver in which a robot flies to a vantage point and lands to collect intelligence. A flying robot capable of perch-and-stare, equipped with cameras and other sensors, can provide an inconspicuous reconnaissance platform. Additionally, robot perching provides a potential means for recharging the robot's power source (e.g., through the use of photovoltaics).

It is desirable for perch-and-stare applications that a robot be able to land on a variety of surfaces. Inspired by the adaptability of songbirds (Order Passeriformes), we present the design of a landing mechanism capable of perching on curved surfaces. Our design is developed with the intention of being attached to a rotorcraft capable of vertical take-off and landing, similar to a bird's transition into a hover-like maneuver when perching (Fig. 1).

For songbirds, foot and leg anatomy is important for perching functionality [2]. Birds' feet are underactuated, meaning they have more degrees of freedom than muscles to control them. This feature enables the feet to passively conform to the structure on which they are attempting to perch. Underactuated grippers are a focus of study for many research groups, and designs have been developed that utilize passive mechanical components such as compliant mechanisms and mechanical limits, as well as clever differential transmissions [3]-[8]. This knowledge base, as well as the fact that most songbirds have an anisodactyl toe arrangement with three forward facing toes and one backward facing toe, motivated the design of our gripper.

This work was supported in part by the University of Utah and the National Science Foundation under grant DGE-0654414.

The authors are with the Department of Mechanical Engineering, University of Utah, Salt Lake City, UT 84112, USA. \{courtney.doyle, jake.abbott, mark.minor $\}$ eutah.edu

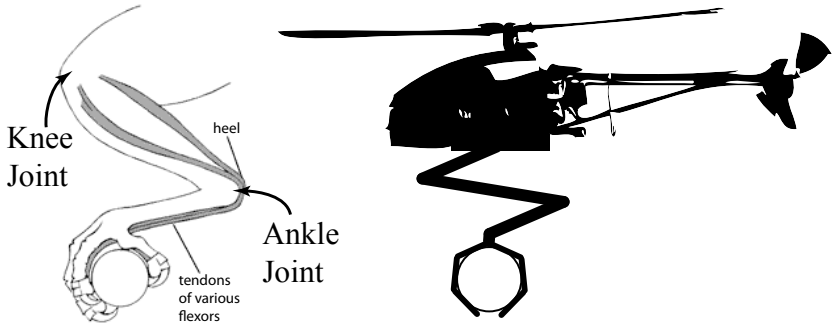

(a)

(b)

Fig. 1. Avian-inspired passive perching. (a) Songbirds have evolved a useful adaptation that allows sleeping while perching. As the bird folds it legs, the tendons on the rear side of the ankle automatically grip the toes around the branch. This enables the relaxed leg of a sleeping bird to tightly grip the perch without any voluntary muscle effort. Image by courtesy of Encyclopaedia Britannica, Inc., copyright 2008; used with permission [1] with added labels. (b) Conceptual drawing depicting a rotorcraft (e.g., helicopter) perching on a curved surface, similar to a bird.

Additionally, songbirds exhibit a particulary interesting adaptation that equips them for life in the trees [2], [9]. As shown in Fig. 1(a), songbirds have a tendon on the rear side of the ankle that automatically causes the toes to grip around a branch when the leg bends. This tendon enables songbirds to sleep while perching without active control of gripping. As a bird relaxes, the weight of its body causes the legs to bend and the toes to grip; deeper relaxation leads to a tighter grip. When the bird is ready to take off, it must actively stand up to let go of the perch. This anatomy is key to the development of our passively actuated system. As depicted in Fig. 1(b), the weight of the rotorcraft is used to actuate the gripping mechanism; when the rotorcraft lifts off, the foot will naturally release.

The usefulness of reliable robot perching is recognized, and a number of research groups are pursuing solutions using a variety of methods. Researchers at the University of Florida and colleagues [10] developed a fixed-wing robot with crawling legs, designed to land on a relatively large target zone, such as the top of a building, and then use its legs to crawl to the edge of the building. Researchers at Drexel University [11] equipped a miniature rotorcraft with optic-flow and ultrasonic sensors, enabling the robot to find and land on the edge of building. Researchers at Cornell University [12] are incorporating a morphing structure inspired by the wings and tail of a bird during landing. Researchers at MIT [13] have developed a method to perform a high-speed maneuver with a fixed-wing robot, such that the robot pulls up into a high angle-of-attack to perch on a string via a latching hook. Researchers at Stanford University [14] have used a 
high angle-of-attack approach to attach to a vertical surface. They use a compliant, bio-inspired leg-type mechanism as a suspension system between the aircraft and foot that uses micro-spines for adhesion. Each of these methods share the need for a specific landing surface for successful perching; the ultimate goal of our avian-inspired approach is to offer perching on the same surfaces available to birds.

To date, a single study has investigated robot grasping inspired by the foot of a bird. Researchers at Clemson University analyzed the feet of raptors, motivated by their excellent stable grasping ability and simple design relative to anthropomorphic hands [15]. They went on to design a nonanthropomorphic "thumbless" grasping hand reminiscent of a raptor foot [16], but most of the joints have independent actuation. Recently, a group at Yale University attached an underactuated hand to the underside of a helicopter [17]. Their work focuses on picking up objects and the effect on flight dynamics, but landing is still accomplished using traditional landing skids. There has been no investigation of bird-feet-inspired graspers for perching.

Our concept is presented as follows. Section II details the development of our design in three parts: II-A discusses the grasping foot; II-B discusses the passively actuated leg; and II-C discusses integration of individual components. Finally, Section III provides performance details of two successful perching trials and discusses future improvements.

\section{MECHANISM DESIGN}

Our perching mechanism was developed as a compilation of two components: grasping via the foot and actuation via the leg. The foot design is an underactuated tendon-driven structure that requires a single actuating force. This actuation force comes from the leg structure, which converts vertical motion of the rotorcraft into tendon displacement. The two components are integrated, creating a passive landing gear.

\section{A. Grasping Foot}

A desirable characteristic of the bird foot is the underactuated structure. This allows the toes to passively conform to unknown surface shapes. Underactuated graspers have been studied intensely, so we drew inspiration from the large body of work for our initial design. Of particular interest to us is an underactuated design of Dollar and Howe in which an 8-dof four-fingered hand is controlled using a single actuator [8]. Their design uses flexible joints between stiff link segments with a cable to transmit actuation force, and is shown to be capable of grasping objects of varying size and shape.

Our foot is a simplified variation on [8]. Using a waterjet, individual toes were cut from a sheet of polyurethane (McMaster-Carr, Item \# 8716K736), using notches to create flexible joints. Hollow tubing was attached to each toe segment for tendon routing. This simple design was chosen for ease of manufacturing and scalability.

Due to the passive actuation method, detailed in the following section, the actuation force is limited by the weight of the rotorcraft. For this reason, friction and joint stiffness are important in the construction of our toes. Friction forces from

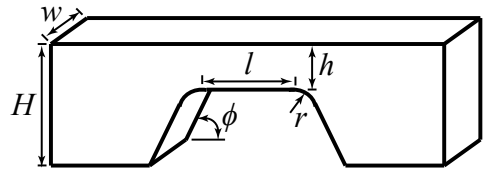

Fig. 2. Parameters characterizing joint geometry. $w$ is the width of the toe, $H$ is the height of the toe, $h$ is the height of the joint, $l$ is the length of the joint, $r$ is the radius of the joint fillet, and $\phi$ is the notch angle.

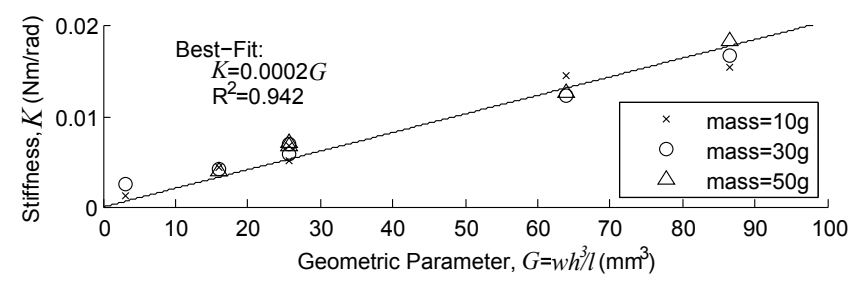

Fig. 3. Experimental data showing that joint stiffness is linearly related to the geometric term $w h^{3} / l$ for the joint shown in Fig. 2.

the tendon reduce the amount of available gripping force; as such, it is should be minimized. For this prototype, friction reduction was performed qualitatively by experimenting with various tendon and tube materials. The materials selected are $12 \mathrm{lb}$ fishing line (Zebco Omniflex monofiliment) and PTFE tubing (McMaster-Carr, Item \# 5239K23). Additionally, locations where the tendon changes direction causes large contact forces, increasing friction. By using curved surfaces to reduce extreme bending, friction is reduced.

In order to study grasping behavior, we developed a model for individual joints and considered kinematic motion of a series of connected toe segments. Each toe joint is characterized by the parameters shown in Fig. 2. Furthermore, each joint can be classified as a small-length flexural pivot and is represented by its pseudo-rigid-body model, Fig. 4(a), as a pin joint with a torsion spring of stiffness $K=E I / l_{0}$, where $E$ is the Young's modulus, $I$ is the cross-section moment of inertia, and $l_{0}$ is the flexible segment length [18].

Because our flexible segment does not have a uniform cross-section due to the notch angle, validation of the model is necessary. By hanging known weights from samples with varying parameters, we were able to investigate the instantaneous center of rotation (ICR) and stiffness of each joint. Though the ICR location varied significantly between samples, it remained relatively stationary for individual joints, validating the pin-joint approximation. Using the relationship $K=M / \theta$, where $M$ is the moment applied to the joint and $\theta$ is the angular deflection of the joint, the stiffness $\mathrm{K}$, which is linearly related to the geometric term $w h^{3} / l$, can be experimentally determined (Fig. 3).

Combining joints in series produces a toe structure that can passively comply to a surface. Development of our toe is based on two central requirements: 1) for the largest workspace, and thus largest range of gripping capability, it is desirable for the joints to deflect in order from proximal to distal; and 2) each joint should be able to return to minimal deflection with no applied tendon tension.

The first requirement can be satisfied by studying the 


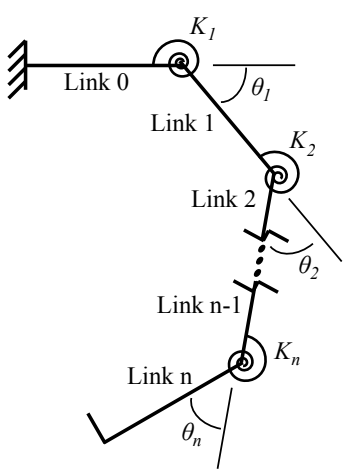

(a)

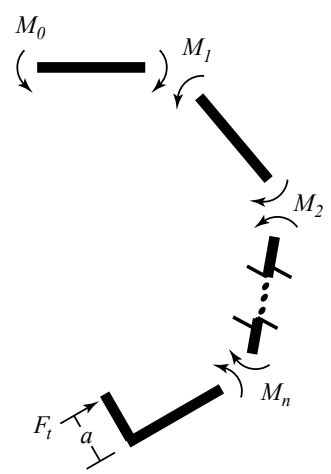

(b)
Fig. 4. Simple model of an $n$-phalanx toe moving through free space. (a) Pseudo-rigid-body model of toe. $K_{n}$ is the joint stiffness of the $n^{t h}$ link and $\theta_{n}$ is the angular displacement of the $n^{\text {th }}$ link. (b) Free body diagram of toe. $M_{n}$ is the moment of the $n^{t h}$ joint, $F_{t}$ is the tendon tension, and $a$ is the distance from rotation to the tendon attachment.

basic model shown in Fig. 4(b). When at static equilibrium, $M_{0}=M_{1}=M_{2}=\ldots=M_{n}=F_{t} a$. Also, because each joint is modeled as a pin/torsion spring mechanism, we know that $M_{n}=K_{n} \theta_{n}$, or equivalently, $\theta_{n}=F_{t} a / K_{n}$. It follows that for a given tension, joints with smaller spring constants will experience larger deflections. Thus, enforcing that $K_{1}<K_{2}<\ldots<K_{n}$ satisfies the first requirement. The importance of having a large workspace is demonstrated in Fig. 5. Notice how the gripping ability changes for each joint pattern. The top row shows a toe with the proposed pattern, $K_{1}<K_{2}<K_{3}$. The toe makes contact with the object starting with the first segment, followed by the second then third, making a successful form closure. The middle row shows a toe with the pattern $K_{1}=K_{2}=K_{3}$. For the perch shown, the toe is able to make a form closure but each segment makes contact at roughly the same time. For an unsymmetrical perch this toe will not be able to grip reliably. The bottom row shows a toe with the pattern $K_{1}>K_{2}>K_{3}$. This toe experiences significant deflection at the third joint first, which causes the toe to miss closure around the perch.

To satisfy the second requirement, we enforce $K_{1}>$ $K_{1 \text { min }}$, where $K_{1 \text { min }}$ is determined by the weight of the distal portion of the toe supported by the first joint. Because the first joint will experience the most weight, and each successive joint is stiffer than the previous, it follows that each joint satisfies the second requirement so long as the first joint does. The above constraints are used to create three identical toes, which are attached to the leg using an adaptation of the anisodactyl arrangement, shown in Fig. 6.

\section{B. Bioinspired Leg Actuator}

Underactuated grippers have been extensively studied, but current solutions require actuation via traditional means such as a motor. For a perch-and-stare application, continuously expending power to hold a grip is undesirable. Possible solutions include the use of a nonbackdrivable system or a locking mechanism, however these would still require power to initiate and release the grip. Our design draws

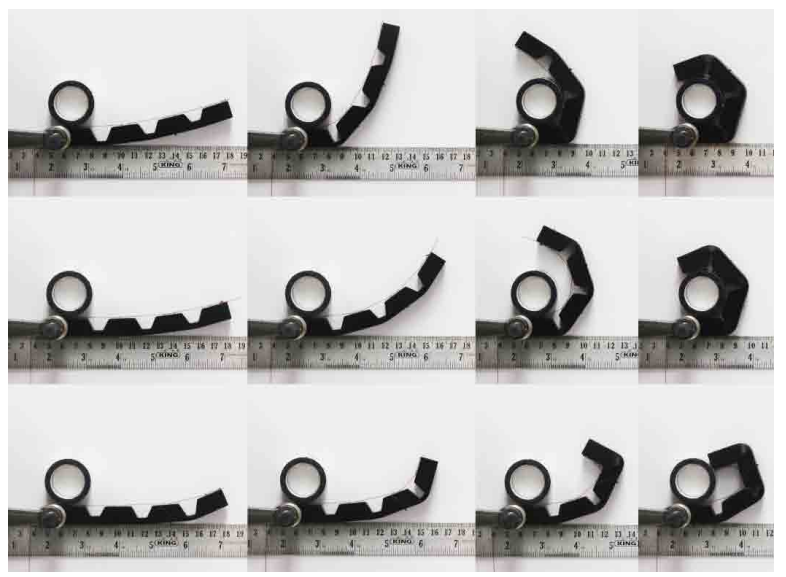

Fig. 5. Depiction of how joint stiffness patterns affect form closure. (Top row): $K_{1}<K_{2}<K_{3}$; (Middle row): $K_{1}=K_{2}=K_{3}$; (Bottom Row): $K_{1}>K_{2}>K_{3}$.

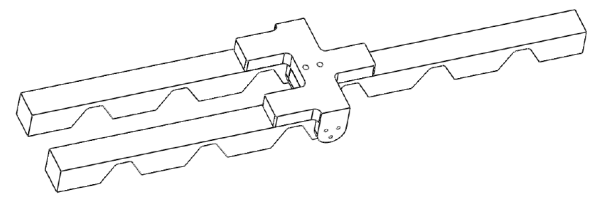

Fig. 6. SolidWorks model of three-toe foot and mounting device.

inspiration from the unique adaptation developed by song birds that enables passive actuation. This bioinspired design must satisfy two requirements: 1) tendon path length must increase as the mechanism collapses; and 2) rotorcraft center of gravity must move in a predictable manner. The first idea is imperative for passive actuation and the second accounts for system stability during the landing maneuver.

Inspired by the single-material design of the toes, we investigated a concept that has evolved into that shown in Fig. 7. This design is mimetic of a bird's leg, consisting of an ankle and knee joint. The structure is formed using a waterjet to cut the shape from a sheet of polycarbonate (Laird Plastics), which makes the prototype easy to manufacture.

In order to satisfy the requirement that the tendon path must increase in length while the leg collapses, knee-cap structures were designed at each joint. By covering a compliant segment with a stiff knee-cap, as the joint deflects the knee-cap creates a varying gap length for the tendon to cover. As the tendon requires more length to cover this gap, it will pull at the foot causing it to close. Additionally, the knee-caps provide a gradual curve for tendon routing, which reduces friction at the points where the tendon changes direction.

The flexible joint was made quite thin in order to reduce the force required to collapse the leg. Any energy utilized in this capacity will reduce the amount of energy available for closing the foot and gripping the perch. The stiffness of each joint can be predicted by studying the pseudo-rigid-body model of an initially curved cantilever beam, as presented in [18]. Similar to experiments with the toe, known forces were applied to knee joints of varying geometries and the stiffness is again found to be linearly related to a geometrical 


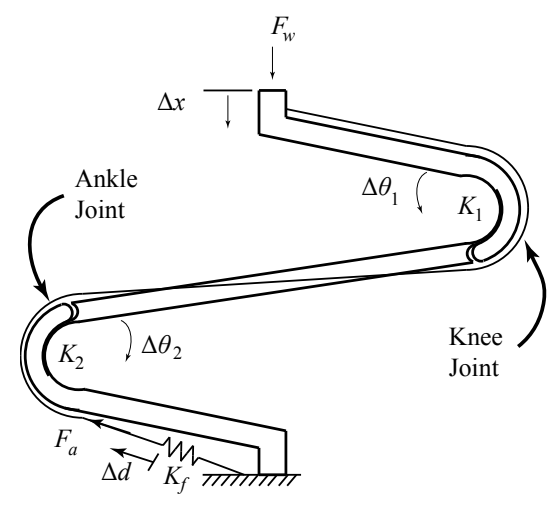

(a)
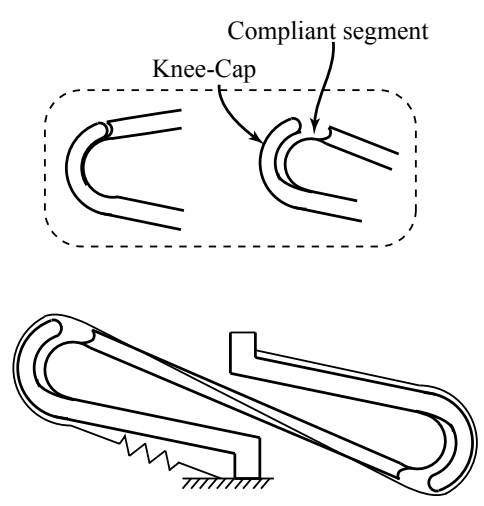

(b)

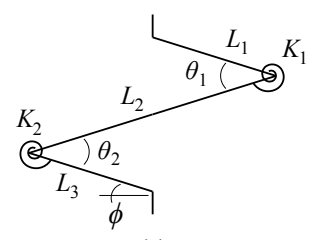

(c)

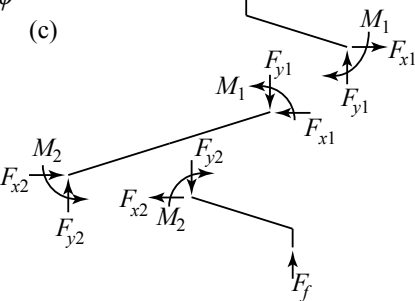

(d)

Fig. 7. Leg mechanism used for passive actuation. (a) Drawing of leg, where $F_{w}$ is the weight of the rotorcraft, $\Delta x$ is the vertical displacement of the rotorcraft, $\Delta \theta_{n}$ is the angular displacement of each leg joint, $\Delta d$ is the linear displacement of the tendon, $F_{a}$ is the actuating tension, $K_{n}$ is the stiffness of each joint, and $K_{f}$ is the effective spring constant of the foot. (b) Leg in collapsed state. Inset depicts the joint functionality. (c) Pseudo-rigid-body model of leg, where $\theta_{n}$ is the angle between leg segments, $L_{n}$ is the length of each leg segment, and $\phi$ is the fixed angle at which the leg leaves the foot. (d) Free body diagram of leg.

term $w h^{3} / l$ where the terms $w, h$, and $l$ are analogous to the parameters depicted in Fig. 2.

For a bird to remain stable while standing or perching, its center of gravity is located directly above, and centered between, the feet [9]. Similarly, the rotorcraft center of gravity should rest directly above the foot of the landing gear. Since the landing gear is designed to perch on a variety of geometries, the tendon will displace by varying amounts, causing the body of the rotorcraft to sit at various heights above the perch. For this reason, it is not sufficient to design a mechanism that only centers the position when fully collapsed; the center of gravity must remain vertically aligned with the foot through the entire motion. Furthermore, the collapsing motion should not exert a moment on the foot that would cause it to move out from under the body.

Design for the latter requirement is achieved by studying the leg model, Figs. 7(c,d); each rigid segment must be in static equilibrium. Balancing forces in the $\mathrm{x}$ and $\mathrm{y}$ directions, $F_{x 1}=F_{x 2}=0$ and $F_{w}=F_{y 1}=F_{y 2}=F_{f}$. Assuming that the leg remains vertically centered, a moment balance of each link produces

$$
L_{1}=\frac{M_{1}}{F_{w} \cos \frac{\theta_{1}}{2}} ; \quad L_{2}=\frac{M_{1}+M_{2}}{F_{w} \cos \frac{\theta_{2}}{2}} ; \quad L_{3}=\frac{M_{2}}{F_{w} \cos \frac{\theta_{2}}{2}} .
$$

Designing the leg such that $K_{1}=K_{2}$, assuming $\theta_{1}=\theta_{2}$, and noting that $M_{1}=M_{2}=K_{1} \theta_{1}$, (1) reduces to constrain the leg links such that

$$
L_{1}=\frac{1}{2} L_{2}=L_{3}
$$

In order to minimize lateral motion of the rotorcraft while perching, a geometric balance is performed. Setting the base of the leg as a zero reference point and using the constraint defined by (2), the horizontal location of the top of the leg is calculated as:

$$
Y_{\text {leg }}=2 L_{1}\left(\cos \left(\theta_{1}-\phi\right)-\cos \phi\right) .
$$

Minimizing (3) over a desirable range of $\theta_{n}$ gives a value of $\phi$ producing the smallest deviation from vertical.

Our design mimics the functionality of a bird's leg and enables passive actuation of an attached tendon-driven gripper. By following the described constraints, the center of mass of an attached rotorcraft will stay vertically centered over the foot. Attaching two symmetric legs to the underside of a rotorcraft should create a platform for stable perching.

\section{Mechanism Integration}

Integrating the foot and leg for successful perching requires a careful consideration of force balance. First, we need to consider the distribution of tendon tension and displacement between the three toes. While unrestricted, each toe should move at the same rate, however if one toe becomes fully constrained by the environment, the remaining toes should be able to continue motion until a complete grip is obtained. Similar to [8], this is achieved through the design of a differential tendon system, shown in Fig. 8. In order to simplify construction, the foot is mounted to the leg such that orientation is opposite of that found in nature; though expected to be minimal, any resulting consequences need to be further examined. The tendon routed from the leg is tied to one side of the pulley mount and the front toe is tied to the opposite side of the mount. The two back toes are connected by a single tendon, routed around the pulley. The tendon in each toe will displace at equivalent rates through open space, providing symmetric closure. If motion in one of the back toes is constrained, the pulley system will allow the remaining toes to move $\Delta d$ and $2 \Delta d$ for the front and back toe, respectively. If the front toe makes contact before the back toes, motion will continue in all three toes until the landing surface is centered appropriately in the foot.

Furthermore, through open space, this setup creates an equal force distribution among each of the toes, such that $F_{1} \approx F_{2} \approx F_{a} / 3$. This is an approximate relationship because the tendon does not necessarily depart the pulley in a 


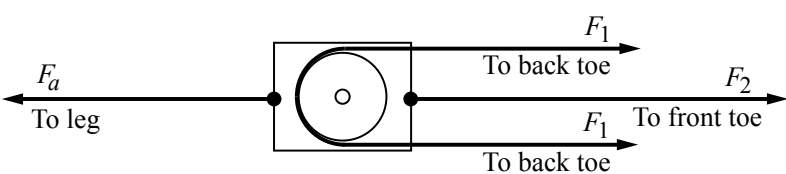

Fig. 8. Differential system applied to tendons, where $F_{1}$ and $F_{2}$ are the tendon tensions in individual toes and $F_{a}$ is the tendon tension applied by the leg. Through open space, each toe moves at equivalent rates. Once one toe is fully constrained by the environment, the remaining toes continue motion until a complete grip is made.

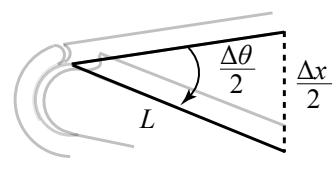

(a)

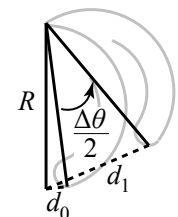

(b)
Fig. 9. Geometrical constraints of the actuating system. (a) Geometrical relationship between $\Delta x$ and $\Delta \theta$, where $L$ is the link length $\left(L_{1}\right)$ and $\Delta \theta$, $\Delta x$ are as defined previously. (b) Geometrical relationship between $\Delta \theta$ and $\Delta d$, where $R$ is the outer knee-cap radius, $d_{i}$ is the tendon path length and $\Delta \theta$ is as defined previously.

direction parallel to $F_{a}$; deviation will cause slight variations in tension. Once motion in one back toe is constrained, the two back toes will still experience equivalent tension, but the tension in the tendon of the back toes will increase at twice the rate of the front toe.

Another important design requirement is that the foot be able to close fully given the available force. The relationship between tension and rotorcraft weight is determined by an energy balance of the system depicted in Fig. 7(a):

$$
F_{w} \Delta x=\frac{1}{2} K_{1} \Delta \theta_{1}^{2}+\frac{1}{2} K_{2} \Delta \theta_{2}^{2}+\frac{1}{2} K_{f} \Delta d^{2} .
$$

By substituting the relationship $F_{a}=K_{f} \Delta d$ and assuming $K_{1}=K_{2}=K$ and $\Delta \theta_{1}=\Delta \theta_{2}=\Delta \theta / 2$, rearranging (4) gives the relationship between the applied tension and the weight of the rotorcraft:

$$
F_{a}=\frac{2 F_{w} \Delta x-K \Delta \theta^{2} / 2}{\Delta d} .
$$

Geometrical relationships exist between $\Delta x, \Delta \theta$, and $\Delta d$, which can be utilized to further characterize the tendon tension. The relationship between $\Delta x$ and $\Delta \theta$ is determined by assuming that the body stays vertically aligned with the foot. This assumption is justified by using the described design process, which causes the body to follow a nearly straight line. In reality, the alignment deviates slightly from vertical and this assumption underestimates the change in angle for a given vertical displacement. Studying Fig. 9(a), the Law of Cosines can be used to show that

$$
\Delta \theta \approx 4 \sin ^{-1}\left(\frac{\Delta x}{4 L}\right) .
$$

The relationship between $\Delta \theta$ and $\Delta d$ is determined by studying Fig. 9(b). Here, $\Delta d / 2=d_{1}-d_{0}$. By making a simplifying assumption that $d_{0}=0$, we can use the Law of Cosines to show that

$$
\Delta d \approx 4 R \sin \left(\frac{\Delta \theta}{4}\right)=\frac{R \Delta x}{L} .
$$

For our system, this assumption introduces less than $10 \%$ error, but does overestimate the amount of tendon displacement for a given change in joint angle. This equation also provides a design metric, as a certain radius is required to achieve a desired amount of tendon travel.

Substituting (6) and (7) into (5) produces an equation that relates the amount of total tension available for actuating the foot and grasping, based on the weight and vertical displacement of the rotorcraft:

$$
F_{a}=\frac{2 L}{R}\left(F_{w}-\frac{2 K\left(\sin ^{-1}\left(\frac{\Delta x}{4 L}\right)\right)^{2}}{\Delta x}\right) .
$$

Due to the simplifying assumptions made in the development of this equation, if $K$ is sufficiently small, this is actually a conservative estimation of the tension, which helps offset nonconservative modeling assumptions (e.g. no friction in tendon routing). The force available for grasping can be determined by subtracting the tension required to overcome toe stiffness in a given configuration from (8).

\section{RESULTS AND DISCUSSION}

A prototype was constructed using the methods described, and is shown in a relaxed state in the left column of Fig. 10. In order to increase the amount of friction at the grip, a soft Buna-N foam (McMaster-Carr, Item \# 93745K21) was attached to the surface of each toe segment. The final prototype weighs $96 \mathrm{~g}$. This prototype was tested on two cylindrical rods with diameters of $49 \mathrm{~mm}$ and $33 \mathrm{~mm}$ by hanging two $100 \mathrm{~g}$ weights on the leg, which was chosen as a convenient weight and is not meant to be representative of rotorcraft weight. Successful perching is demonstrated in the center and right columns of Fig. 10.

Our prototype shows that passive perching on curved surfaces can be achieved using an avian-inspired approach. However, this design is an un-optimized solution, and improvements are currently being investigated. First, although the compliant leg mechanism is desirable for manufacturablity and scalability, at this scale the tension forces are too large and the compliant members in the knee and ankle begin to deflect at undesirable locations. This causes a decrease in performance, as extra energy in the system acts to deform these members rather than increasing tension. The deformation creates significant stresses, which cause the compliant members to fatigue prematurely. The choice of polycarbonate for the flexure also contributes to fatigue problems. Second, the lateral stability of the leg is weak due to the long, thin members, which will reduce stability in the whole system under reasonable disturbances.

Currently, in order to work around these difficulties, the toes are designed such that a complete grip is possible at very low tension, so that the leg joints do not deform significantly. This design consideration directly corresponds 


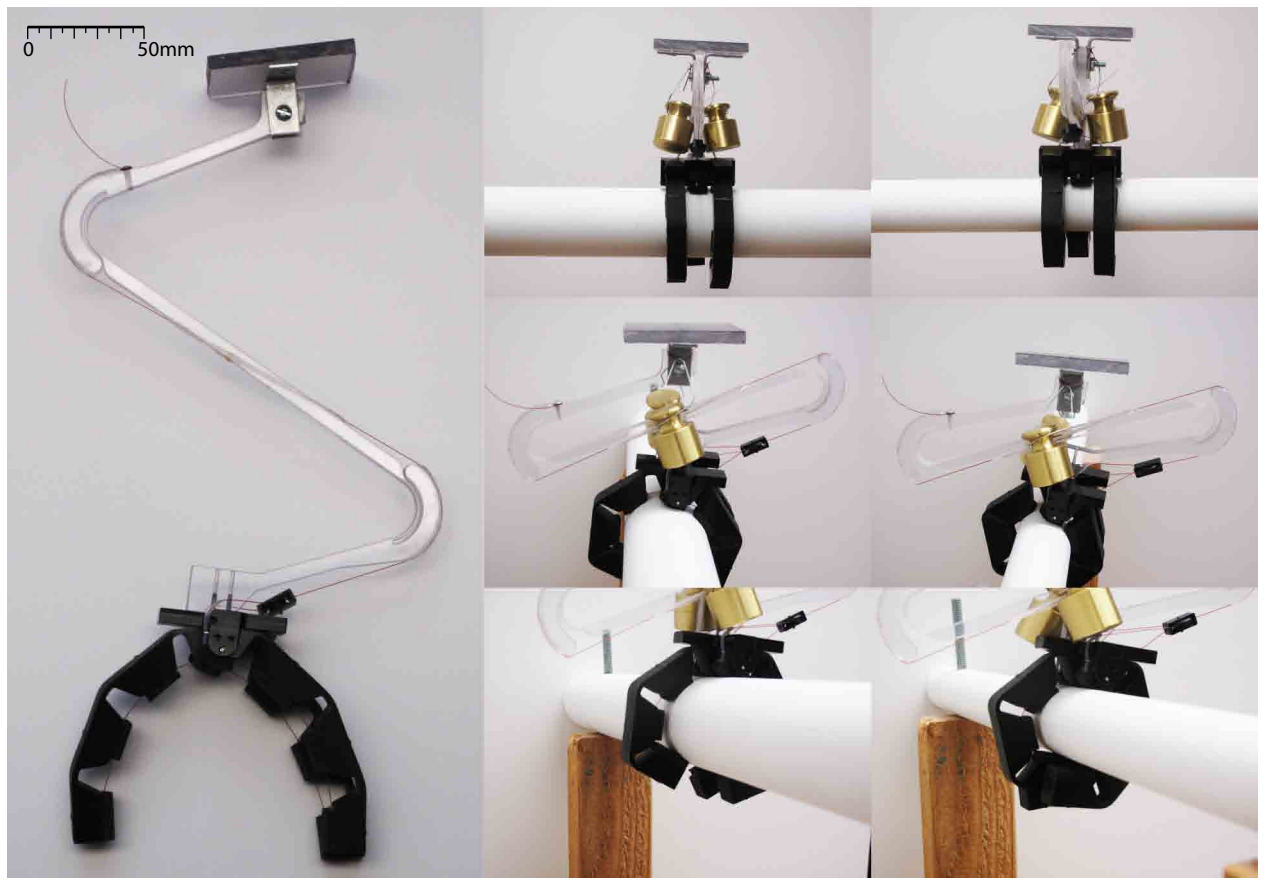

Fig. 10. Demonstration of functional prototype. (Left column): Image of landing gear in its relaxed state. (Center column): Series of images showing a successful perch on a rod of diameter of $49 \mathrm{~mm}$. (Right column): Series of images showing a successful perch on a rod of diameter of $33 \mathrm{~mm}$.

to weak compliant members at each toe joint, which means the toes sag under their own weight when the system is relaxed. Methods currently under consideration to reduce sag include adding a pre-curve to the toe (similar to the blades of a helicopter or wings of a plane) and selecting lighterweight materials. Additionally, a grip-force analysis is being conducted so that we can predict the ability of the system to resist environmental disturbances while perched.

\section{CONCLUSIONS}

This paper presents a prototype that demonstrates the concept of a passive, avian-inspired perching mechanism. Successful perches were made and were stable under small disturbances. Our results show promise that future iterations should perform well as a solution for passive, compliant landing gear for perch-and-stare applications on curved surfaces.

\section{ACKNOWLEDGMENT}

The authors would like to acknowledge the support of Tom Slowik in the Department of Mechanical Engineering and Laird Plastics. Additionally, the authors would like to thank Dr. Ron Meyers at Weber State University for fruitful discussions about bird anatomy and function.

\section{REFERENCES}

[1] E. Britannica. (2011) Bird. [Online]. Available: http://www.britannica.com/EBchecked/topic/66391/bird

[2] N. S. Proctor and P. J. Lynch, Manual of Ornithology: Avian Structure and Function. Ann Arbor, Michigan: Edwards Brothers, Inc., 1993.

[3] J. D. Crisman, C. Kanojia, and I. Zeid, "Graspar: A flexible, easily controllable robotic hand," IEEE Robotics and Automations Mag., vol. 3, no. 2, pp. 32-38, 1996.

[4] B. Massa, S. Roccella, M. C. Carrozza, and P. Dario, "Design and development of an underactuated prosthetic hand," in Proc. IEEE Int. Conf. Robotics and Automation, 2002, pp. 3374-3379.
[5] T. Laliberté, L. Birglen, and C. M. Gosselin, "Underactuation in robotic grasping hands," Machine Intelligence and Robotic Control, vol. 4, no. 3, pp. 1-11, 2002.

[6] L. Birglen and C. M. Gosselin, "Kinetostatic analysis of underactuated fingers," IEEE Trans. Robotics and Automation, vol. 20, no. 2, pp. 211-221, 2004.

[7] C. Gosselin, F. Pelletier, and T. Laliberté, "An anthropomorphic underactuated robotic hand with 15 dofs and a single actuator," in Proc. IEEE Int. Conf. Robotics and Automation, 2008, pp. 749-754.

[8] A. M. Dollar and R. D. Howe, "The highly adaptive SDM hand: Design and performance evaluation," Int. J. Robotics Research, vol. 29, pp. 585-597, 2010.

[9] F. B. Gill, Ornithology, 3rd ed. New York: W. H. Freeman and Company, 2007.

[10] R. J. Bachmann, F. J. Boria, R. Vaidyanathan, P. G. Ifju, and R. D. Quinn, "A biologically inspired micro-vehicle capable of aerial and terrestrial locomotion," Mechanism and Machine Theory, vol. 44, pp. 513-526, 2009

[11] T. W. Danko, A. Kellas, and P. Y. Oh, "Robotic rotorcraft and perchand-stare: Sensing landing zones and handling obscurants," in Proc. IEEE Int. Conf. Advanced Robotics, 2005, pp. 296-302.

[12] A. M. Wickenheiser and E. Garcia, "Optimization of perching maneuvers through vehicle morphing," J. Guidance, Control, and Dynamics, vol. 31 , no. 4 , pp. 815-823, 2008.

[13] R. Cory and R. Tedrake, "Experiments in fixed-wing UAV perching," in Proc. AIAA Guidance, Navigation, and Control Conf., 2008, pp. $1-12$.

[14] A. L. Desbiens, A. T. Asbeck, and M. R. Cutkosky, "Landing, perching and taking off from vertical surfaces," Int. J. Robotics Research, vol. 30, no. 3, pp. 355-370, 2011.

[15] A. M. Ramos and I. D. Walker, "Raptors-inroads to multifingered grasping," in Proc. IEEE/RSJ Int. Conf. Intelligent Robots and Systems, 1998, pp. 467-475.

[16] A. M. Ramos, I. A. Gravagne, and I. D. Walker, "Goldfinger: A non-anthropomorphic, dextrous robot hand," in Proc. IEEE Int. Conf. Robotics and Automation, 1999, pp. 913-919.

[17] P. E. I. Pounds and A. M. Dollar, "Hovering stability of helicopters with elastic constraints," in Proc. ASME Dynamics Systems and Control Conf., 2010.

[18] L. L. Howell, Compliant Mechanisms, 1st ed. New York: John Wiley \& Sons, Inc., 2001. 